\title{
Wheel loader operation-Optimal control compared to real drive experience
}

\author{
Vaheed Nezhadali, B. Frank and Lars Eriksson
}

\section{Linköping University Post Print}

\section{Tweet}

N.B.: When citing this work, cite the original article.

Original Publication:

Vaheed Nezhadali, B. Frank and Lars Eriksson, Wheel loader operation-Optimal control compared to real drive experience, 2016, Control Engineering Practice, (48), 1-9.

http://dx.doi.org/10.1016/j.conengprac.2015.12.015

Copyright: Elsevier

$$
\text { http://www.elsevier.com/ }
$$

Postprint available at: Linköping University Electronic Press

http://urn.kb.se/resolve?urn=urn:nbn:se:liu:diva-126245

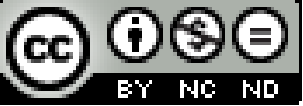




\title{
Wheel loader operation-Optimal control compared to real drive experience
}

\author{
V. Nezhadali ${ }^{\mathrm{a}}$, B. Frank ${ }^{\mathrm{b}}$, L. Eriksson ${ }^{\mathrm{c}}$ \\ ${ }^{a}$ Vehicular Systems, Electrical Engineering Department, Linköping University, SE-581 83 Linköping, Sweden \\ (e-mail: vaheed.nezhadali@liu.se,Tel: +4613-281327; fax: +4613-139282) \\ ${ }^{b}$ Faculty of Engineering, Lund University, 118 S-221 Lund, Sweden - Volvo Construction Equipment, SE-631 85, Eskilstuna, Sweden \\ (e-mail: bobbie.frank@volvo.com) \\ ${ }^{c}$ Vehicular Systems, Electrical Engineering Department, Linköping University, SE-58183 Linköping, Sweden (e-mail: larer@isy.liu.se)
}

\begin{abstract}
Wheel loader trajectories between loading and unloading positions in a repetitive loading cycle are studied. A wheel loader model available in the literature is improved for better fuel estimation and optimal control problems are formulated and solved using it. The optimization results are analyzed in a side to side comparison with measurement data from a real world application. It is shown that the trajectory properties affect the operation productivity. However, efficient trajectories are not the only requirement for high productivity operation and all major power consuming sources such as vehicle dynamics, lifting and steering have to be included in the optimization for productivity analysis. The effect of operator steering capability is also analyzed showing that development of autonomous vehicles can be envisaged especially for repetitive cycles.
\end{abstract}

Keywords:

Optimal control, modeling for control, powertrain modeling and simulation, trajectory optimization

$\begin{array}{llll}\text { Nomencalture } & M_{b u c} & \text { Mass of load in the bucket } \\ \omega_{e} & \text { System state, Engine speed } & H_{b u c} & \text { Bucket height } \\ p_{i m} & \text { System state, Intake manifold pressure } & \theta_{1} & \text { Bent angle of lift arm } \\ \omega & \text { System state, Angular speed of lift arm } & F_{c y l} & \text { Lift force } \\ \theta & \text { System state, Angle between lift arm and horizontal axes } & \alpha & \text { Angle between lift force and lift arm } \\ V & \text { System state, Vehicle speed } & r_{1}, r_{2}, R & \text { Lift arm dimensions } \\ X & \text { System state, WL position } & y_{g} & \text { Height of the hinge between body and lift arm } \\ Y & \text { System state, WL position } & F_{a r m, w} & \text { Lift arm weight } \\ \beta & \text { System state, Heading angle of WL } & Q & \text { Mass flow rate into lift cylinders } \\ \delta & \text { System state, Steering angle } & A_{l c} & \text { Lift cylinder cross section area } \\ u_{f} & \text { Control input, Fuel mass injected per combustion cycle } & n_{l c} & \text { Number of lift cylinders } \\ u_{p} & \text { Control input, Lift cylinder pressure } & \eta_{l i f t} & \text { Lift system efficiency } \\ u_{s} & \text { Control input, Steering angle time derivative } & \phi & \text { Speed ratio over torque converter } \\ u_{b} & \text { Control input, Braking force } & \gamma & \text { Gear ratio } \\ J_{e} & \text { Engine inertia } & r_{w} & \text { Wheel radius } \\ P_{e, l o a d} & \text { Engine load } & P_{p u m p} & \text { Power on engine side of torque converter } \\ T_{e} & \text { Engine torque } & P_{t u r b} & \text { Power on wheel side of torque converter } \\ P_{l i f t} & \text { Lifting power } & \eta_{T C} & \text { Torque converter efficiency } \\ P_{s t e e r} & \text { Steering power } & c_{s t} & \text { Steering load constant } \\ P_{t r a c} & \text { Traction power } & T & \text { Short loading cycle duration }\end{array}$

$F_{w} \quad$ Traction force at wheels

$\tau_{p} \quad$ Time constant in pressure dynamics

$p_{\text {stat }} \quad$ Stationary intake manifold pressure

$F_{\text {roll }} \quad$ Rolling resistance force

$M_{\text {tot }} \quad$ Mass of WL+load+rotating inertia equivalent

$T_{b u c} \quad$ Torque on lift arm due to bucket load

$T_{\text {arm,w }} \quad$ Torque on lift arm due to its own weight

$R_{\text {turn }} \quad$ WL turning radius

$m_{f} \quad$ Fuel mass

$\rho_{f} \quad$ Fuel density

$F_{b u c} \quad$ Bucket and load weight

\section{Introduction}

Wheel loaders (WL) are categorized as construction machines with frequent application in mining and other construction environments. Due to the fact that the capacity of a WL bucket is limited and usually smaller than the total amount of load to be displaced, WL loading and unloading operation is repeated several times. In such high frequency application, investigating how fast WLs can perform a loading operation or how much fuel can be saved during the operation is a common point of interest for both WL owners and manufacturers. The 
productivity of WL operation can be described according to the fuel consumption and operation duration for transfer of certain load while lower values of both time and fuel correspond to higher productivity. However these two objectives are contradictory and minimizing one, results in the increase of the other, thus encouraging to obtain an efficient compromise between the two. The analysis of different solutions to increase WL productivity can be performed via different experimental operations and measurements or by mathematical optimization of suitable WL models. Since performing measurements with WLs is by far more costly and time consuming, manufacturers favor methods which can replace the measurements yet producing reliable results.

Short loading cycle (SLC), is a typical operating cycle for WLs and is illustrated in Figure 1. WL loading cycles are highly transient operations during which various components in steering, lifting, and powertrain subsystems interact to perform the loading process while operating in different ranges of efficiency. There are also workplace parameters such as the placement of the load receiver with respect to the WL, different loading conditions at each loading occasion or the road surface condition which add up to the size of the optimization problem when analyzing WL operation efficiency. This paper presents an example where such optimization problems can be solved using optimal control (OC) while the implemented methodology and the results are insightful considering the growing interest for development of autonomous vehicles or operator assist systems, $<1>$.

Different studies are carried out for quantification, control and simulation of various subsystem properties and dynamics during the WL operation. In $<2>$ the focus is only on the lift hydraulics and linkage dynamics and a controller is designed for bucket leveling. In $<3>$ optimized engine transients for fuel efficient operation are calculated without including the lifting and steering dynamics. Efficient operator and machine interactions during WL operation are analyzed in $<4$; $5>$ with emphasis only on the influence of the human operator in the dynamic simulations. A WL model including steering and lifting hydraulics while representing the diesel engine with an electric motor is presented in $<6>$ aiming at powertrain controller evaluation. In $<7 ; 8>$ modeling, simulation and control are at the center with no trajectory optimization in the loop. Geometrical analysis of optimal WL trajectories are also performed in numerous works as $<9 ; 10 ; 11 ; 12 ; 13>$ where the diesel engine and lifting dynamics are not included. The contribution in this work is that in addition to including major dynamics of diesel engine, lifting hydraulics and steering system in the model, trajectory planning and optimization of the complete system transients are also considered in the analysis of WL operation in the SLC.

The novelty in this paper is that a WL model available in the literature $<14>$ is improved such that despite the nonlinear properties of certain components and the presence of discontinuous gear shifts during the WL operation, it can acceptably predict fuel consumption and component transients during a loading cycle and more importantly is compatible with optimal control problem formulation requirements. The key contribution is

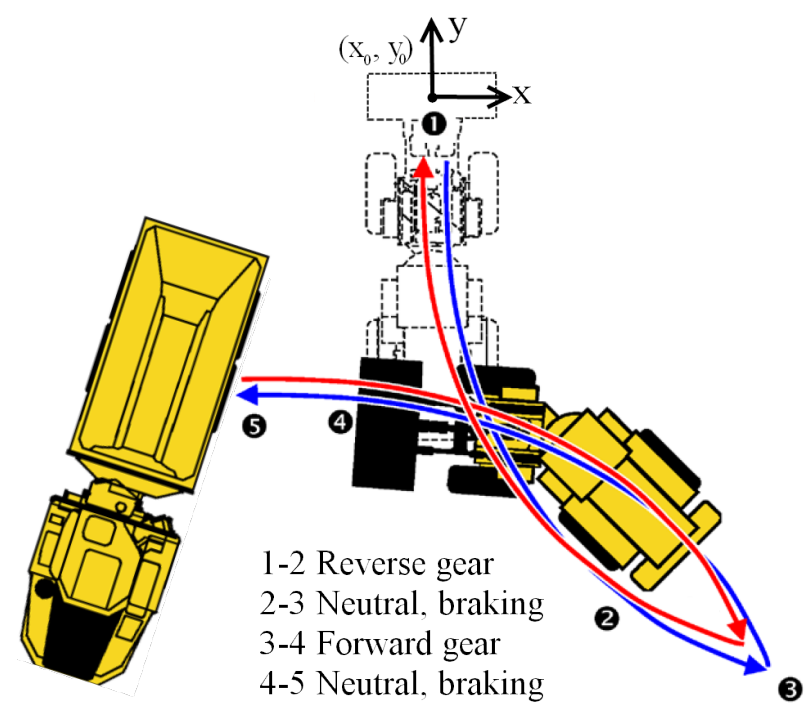

Figure 1: Typical WL trajectory and choice of gears in a SLC operation. Point 3 will be referred to as reversing point since WL moving direction switches from backward to forward at this point. Picture from $<9>$

a side to side comparison of measured WL trajectories and results from OC while showing how OC can be used to analyze and improve the performance in such industrial applications.

\subsection{Paper outline}

Section 2 of the paper describes the details of the measurement setup and how the SLC operation is performed by operators of different skill level while component transients are measured. The measurements are later used to define the boundary values for the OC problem formulation. First in Section 3 new models are developed and parametrized for diesel engine, lifting hydraulics and torque converter (TC) such that the complete WL model can fairly well approximate the measured fuel consumptions when following the measured speed, lifting, steering and WL trajectories.

Then in Section 3, OC problems representing the SLC properties such as payload and driving distance similar to the measurements are formulated and solved using the developed model.

The results from optimization and measurements are compared and analyzed in Section 4. Candidate cycles from measurements are selected and path constraints are defined in the OC problem formulation such that the same trajectories are followed while optimizing the rest of transients. Also, for these sub-optimal trajectories the trade-off between fuel and time objectives is calculated and compared to the OC obtained tradeoffs. Finally, the effect of operators' capability in fast WL steering on the WL trajectory and operation productivity is investigated.

\section{Measurement setup}

To investigate the potential increase in fuel efficiency and/or productivity by using OC, the SLC of loading gravel onto a load 


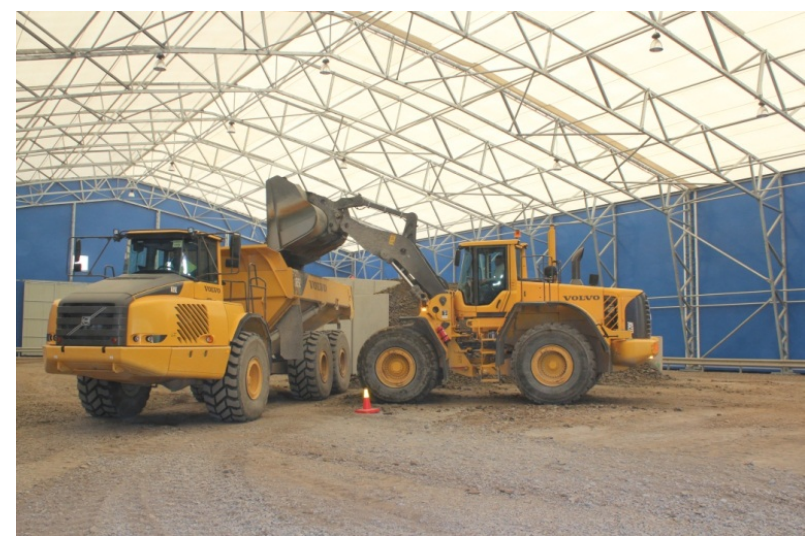

Figure 2: SLC measurement setup, loading gravel onto an articulated hauler inside a tent.

receiver is chosen, i.e a common application of a larger production chain including a WL. The chosen loading operation is an example of a typical re-handling application where processed material has been stock-piled and a WL loads it onto out-going trucks. To be able to compare the OC results with the measurements it is important to isolate the operator behavior as the sole source of deviations $<15>$. This is done by using the same machine with the same equipment and same bucket and same tires for all operators to minimize the machine specification dependence and using the same calibrated gravel pile to minimize the working environment dependence. The same gravel is reused for all operators, in the hope that it would minimize the deviation in bucket fill easiness, differences in fuel efficiency, and productivity dependence on the material and environment. Also the road surface is smoothed after every operator so that all operators have the same preconditions. To further decrease the working environment dependence, the measurements are conducted inside a tent, see Figure 2, therefore the measured data is unaffected by the weather condition.

The worksite is set up according to Figure 1, where the hauler position is fixed to roughly 30 degrees angle from the normal of the gravel pile. However the reversing point in Figure 1 is decided by the WL operator, and also the shape of the gravel pile changes after every loading cycle where some parts are removed and therefore the loading point varies for different measured cycles. Using a Volvo L220F WL $<16>58$ operators of various skills, as follows, are included in the study to have a wide range of measured results when comparing with the OC solutions. Every operator performs 4 loading cycles while sensors are placed on the WL to measure different signals. The skill groups are:

1. Operators that know how a WL works but do not operate WL as a profession.

2. Operators that are evaluating WL and/or working as test operators and/or show operators and/or trainers at Volvo.

3. Operators that are working in every day WL bucket applications in production chains as a profession.

Additionally, three of the most experienced internal test operators are asked to do the so called intensity measurements, meaning that they were supposed to operate the WL in three different intensities as follows:

1. Slow driving and low bucket fill factor, corresponding to Sunday driving.

2. Medium driving pace and medium bucket fill factor, corresponding to what to expect when operating the WL in 8 hour shifts.

3. As fast possible driving and as full bucket as possible, corresponding to a pace that only could be held by an operator for less than one hour due to the high mental and physical workload on the operator.

The reason for these intensity measurements is to map the complete WL working area in respect to productivity versus fuel efficiency. All other operators are asked to operate the WL in a pace corresponding to how they would work if they were supposed to do an 8 hour shift with the specified work assignment. All signals, except steering angle $\delta$ due to technical issues, are measured via the internal CANbus in the machine at a sampling rate of $50[\mathrm{~Hz}]$ while some unexpected issues are also encountered during the measurements. The material is worn a little bit more than expected resulting in finer material than usual causing a higher density of gravel for operations closer to the end of the measurements. This results in that it is somewhat easier to get a heavier load in the bucket at the end of the measurement, hence also easier to get higher productivity and fuel efficiency. Detailed analysis of the measurement results including the comparison between performance of different operators and their so called productivity is presented in $<15>$.

The WL trajectories in Figure 3-right are calculated using the signals from vehicle speed and steering angle sensors. According to the steering system model presented in $<14>$ and knowing $\delta$ from the measurement and WL wheelbase $(L)$ form $<16>$, the turning radius is calculated by:

$$
R_{\text {turn }}=\frac{L}{2 \tan (\delta / 2)}
$$

As will be described in the next section, vehicle heading angle $\beta$ is obtained from (10) and used in (8) and (9) to calculated the $X$ and $Y$ coordinates, respectively. This approach is used since measuring with a GPS, the accuracy is only within 2 [m] while using the internal signals and the articulated hauler position at unloading point as reference, the absolute position estimation fault remains within $1[\mathrm{dm}]$ during the transport phases.

As seen in Figure 3-right and mentioned earlier, the starting point varies from cycle to cycle due to deformation of the gravel pile. Moreover, WL operators select loading points and steering maneuvers according to their ability at aiming for a specific position or their anticipation about the best trajectory. The productivity of the measured operations is also presented in Figure 3-left while later in Section 4 these results are analyzed and then selected WL trajectories are compared with the results from OC. In order to account for the amount of transferred load during the operation, instead of comparing different cases based on only cycle duration and fuel consumption, the data is plotted in [Transferred load / Consumed fuel][Transferred load / Cycle duration] plane. 


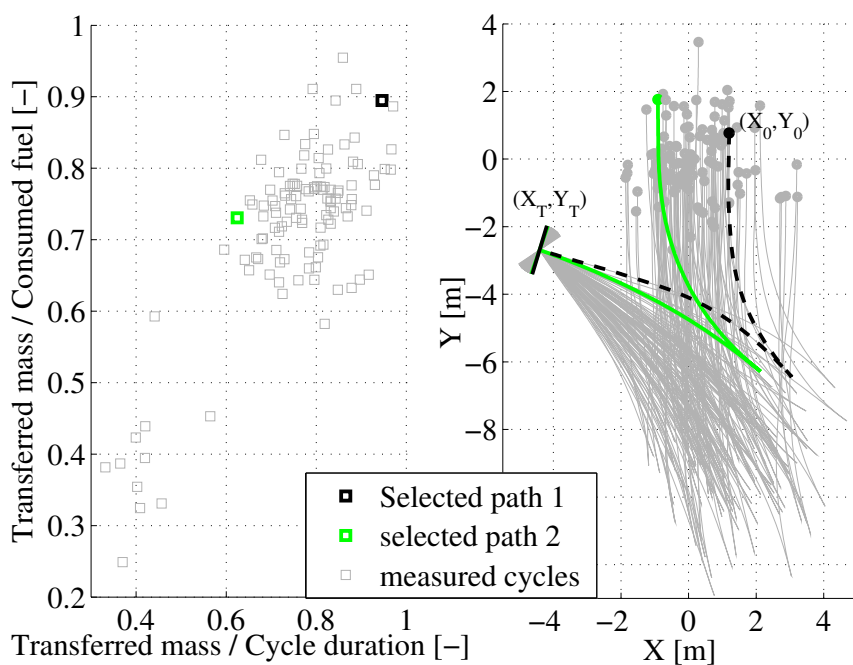

Figure 3: Left: Calculated productivity for measured SLC operations where values are normalized with respect to maximums in this figure. Right: Recorded WL trajectories during measurements. The selected trajectories have almost same traveling distance and bucket load but are very different in productivity. Solid circles show start of the trajectory at $\mathrm{t}=0$ and corresponding load receiver orientation at the unloading point, $\mathrm{t}=\mathrm{T}$, is shown by the perpendicular line at the end of the trajectories.

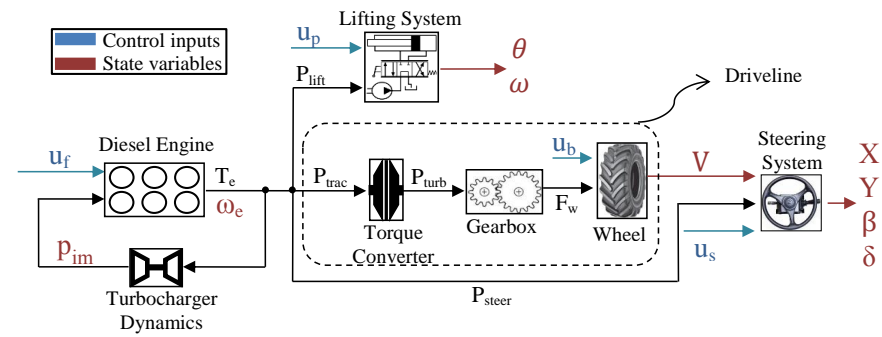

Figure 4: WL model and the interconnection between subsystems. Diesel engine generates power for lifting, transmission and steering.

\section{Optimal control setup}

\subsection{Modeling for optimal control}

The WL model is going to be utilized for OC, therefore it is important to ensure that all utilized functions in the model are continuously differentiable and do not introduce any discontinuity for the range of state and control input variables corresponding to the WL application. Lifting and steering subsystems and the driveline - that comprises of a Torque Converter(TC), gearbox and longitudinal vehicle dynamics - are the three power consumers in a WL, while a diesel engine generates the required power for all the components. Figure 4 shows the overview of the WL model and the interconnection between various subsystems and components while the following general assumptions and simplifications are made in WL modeling:

1. The power required for auxiliary units and tilt function of the bucket are neglected.

2. Constant efficiency is considered for hydraulic lifting pumps and gearbox.
3. A constant rolling resistance for the road surface is assumed while lateral tire friction and resistive aerodynamic forces are neglected.

4. Steering hydraulics is not included in the model for simplicity.

5. Connection between power source, diesel engine, and power consumers is assumed to be loss-free.

6. Lift arm is simplified as a bent slab with constant width and thickness over its length.

Although a basic WL model was already developed in $<14>$, in order to have better agreement between the measurements and the model, new models are developed for engine, lifting system and TC which will be presented in the next sections. The complete WL model has nine states $x$ and four control inputs $u$, see the nomenclature for a description of the variables, which are:

$$
\begin{aligned}
& x=\left[\omega_{e}, p_{i m}, \omega, \theta, V, X, Y, \beta, \delta\right]^{T} \\
& u=\left[u_{f}, u_{p}, u_{b}, u_{s}\right]^{T}
\end{aligned}
$$

and the state dynamics are described by differential equations as the following:

$$
\begin{aligned}
\frac{d \omega_{e}}{d t} & =\frac{1}{J_{e}}\left(T_{e}\left(\omega_{e}, p_{\text {im }}, u_{f}\right)-\frac{P_{e, \text { load }}\left(\omega_{e}, \omega, V, u_{p}, u_{s}\right)}{\omega_{e}}\right) \\
\frac{d p_{\text {im }}}{d t} & =\frac{1}{\tau_{p}\left(\omega_{e}\right)}\left(p_{\text {stat }}\left(\omega_{e}, u_{f}\right)-p_{\text {im }}\right) \\
\frac{d \omega}{d t} & =\frac{F_{\text {cyl }}\left(u_{p}\right) R \sin (\alpha(\theta))-T_{\text {buc }}(\theta)-T_{\text {arm }, w}(\theta)}{I_{\text {boom }}} \\
\frac{d \theta}{d t} & =\omega \\
\frac{d V}{d t} & =\frac{F_{w}\left(\omega_{e}, V\right)-\operatorname{sign}(V)\left(u_{b}+F_{\text {roll }}\right)}{M_{\text {tot }}} \\
\frac{d X}{d t} & =V \cos (\beta) \\
\frac{d Y}{d t} & =V \sin (\beta) \\
\frac{d \beta}{d t} & =\frac{V}{R_{\text {turn }}(\delta)} \\
\frac{d \delta}{d t} & =u_{s}
\end{aligned}
$$

The functions $P_{e, l o a d}, p_{s t a t}, T_{b u c}, F_{c y l}$ and $T_{a r m, w}$ are described in the following sections of the paper.

\subsubsection{Engine model}

The engine output shaft is connected to the TC input transferring the power required for vehicle traction to the wheels. For load lifting and steering purposes, hydraulic pumps are also mechanically coupled to the engine.

A mean value engine model with the same structure as in $<3>$ is used here but it is parametrized according to the available measured data for the engine of a L220F Volvo WL $<16>$. The control input to the engine model is $u_{f}$ which generates the 
engine torque $T_{e}$ considering engine friction and turbocharger dynamics. Engine speed and intake manifold pressure are the engine state variables with the dynamics given by (3) and (4) where $p_{\text {stat }}$ is the parametrized stationary intake manifold pressure map and $\tau_{p}$ is the engine speed dependent time constant as described in $<3>$. The engine load is calculated as the sum of powers required for lifting, steering and traction as:

$$
P_{e, \text { load }}=P_{\text {lift }}\left(\omega, u_{p}\right)+P_{\text {steer }}\left(u_{s}\right)+P_{\text {trac }}\left(\omega_{e}, V\right)
$$

and then using $T_{e}$ and the Newton's second law in (3) the engine speed dynamics are obtained. The mass of injected fuel in [gr] for a cycle of length $\mathrm{T}$ [sec] is calculated by:

$$
m_{f}=\int_{0}^{T} \frac{6 u_{f} \omega_{e}}{4 \pi \rho_{f}} d t
$$

\subsubsection{Lifting subsystem}

To calculate the kinematics of the bucket lifting arm, the geometry of the arm is studied. Figure 5 shows the geometry of the arm where variable and fixed dimensions during lifting are illustrated by dashed and solid lines respectively. Hydraulic force from the two lift cylinders should overcome the weight of the load and bucket arm for upward movement of the arm. Fluid pressure in the lift cylinders $u_{p}$ generates the required hydraulic force and it is considered as the control input to the lifting subsystem. The resulting angular acceleration of the bucket arm is therefore calculated by the following:

$$
\begin{gathered}
T_{b u c}=F_{b u c}\left(r_{1} \cos (\theta)+r_{1} \cos \left(\theta-\theta_{1}\right)\right) \\
T_{a r m, w}=F_{a r m, w} r_{1} \cos (\theta)
\end{gathered}
$$

The angular velocity of the bucket arm is then calculated by (5) where $\alpha(\theta)$ is depicted in Figure 5, and the lift force is calculated by:

$$
F_{c y l}=n_{l c} u_{p} A_{l c}
$$

The height at the end of the arm is obtained according to the arm geometry by:

$$
H_{b u c}=\left(r_{1}^{2}+r_{2}^{2}-2 r_{1} r_{2} \cos \left(\theta-\theta_{1}\right)\right) \sin (\theta)+y_{g}
$$

where $\theta$ is calculated from (6).

Using the piston displacement speed in the lift cylinders at the connecting point of lift cylinders and lift arm, the mass flow rate from lifting pumps is calculated:

$$
Q=R \omega \sin (\alpha) A_{l c} n_{l c}
$$

The lifting power acting as a part of the engine load is calculated as:

$$
P_{l i f t}=\max \left(0, \frac{Q u_{p}}{\eta_{l i f t}}\right)
$$

where the max represents the hydraulic valves enabling bucket holding or lowering without using engine power.

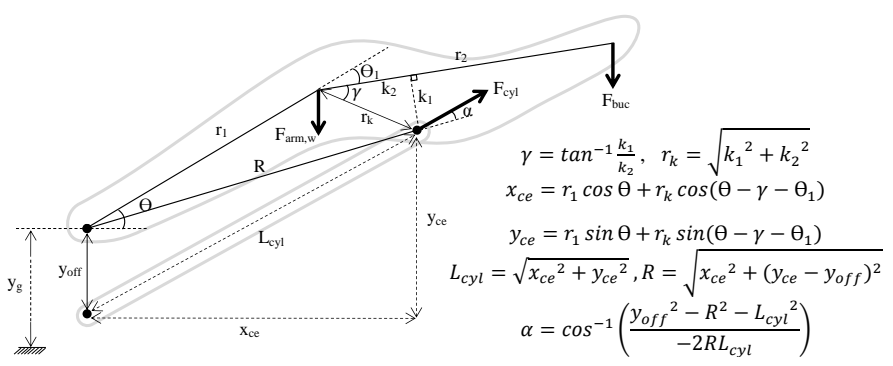

Figure 5: Lift arm geometry and acting forces on the arm during load lifting are considered in order to calculate lift kinematics.

\subsubsection{Driveline}

A Torque Converter (TC) transfers the torque from the engine to the wheels and in the reverse direction. The direction is determined by the speed ratio $\phi=\frac{\gamma V / r_{w}}{\omega_{e}}$ between output and input shafts of the TC such that while the pumping side of the TC rotates faster than the turbine side connected to the gearbox $(\phi<1)$, power is transferred to the wheels. When the turbine side rotates faster than the pumping side $(\phi>1)$, the power is transferred in the reverse direction meaning that the kinetic energy at wheels becomes available for load lifting or engine acceleration however TC efficiency reduces to almost one third of its normal operation value while operating in the reverse mode.

The typical approach for TC modeling with control purposes, presented in $\langle 17\rangle$, is to use the two TC characteristic curves one for torque multiplication and another for the generated torque on pumping side of TC for different speed ratios. This however becomes tricky when modeling for optimal control because modeling the reversing phenomena in the TC introduces a large discontinuity in the torque multiplication curve at the reversing point when there exist no lock-up function. Using the approach mentioned in $<14>$ to remove the discontinuities would still produce short intervals of larger than one efficiency at the reversing point which is not practical. Therefore a new approach is implemented where TC efficiency is used instead of torque multiplication factor. Power at TC input and output is then modeled using the speed ratio dependent efficiency $\eta_{T C}$ and generated torque on pumping side $T_{\text {pump }}$ as:

$$
\begin{aligned}
& P_{\text {pump }}=T_{\text {pump }}(\phi) \omega_{e}\left(\frac{\omega_{e}}{1000}\right)^{T_{1}} \\
& P_{\text {turb }}=P_{\text {pump }} \eta_{T C}(\phi)\left(\frac{\omega_{e}}{1000}\right)^{T_{2}}
\end{aligned}
$$

where $\omega_{e}$ is in $[\mathrm{rpm}], T_{1}$ and $T_{2}$ are the tuning parameters and $T_{\text {pump }}$ and $\eta_{T C}$ are curve fitted to available TC data at $\omega_{e}=1000[\mathrm{rpm}]$ shown in Figure 6.

Vehicle speed dynamics is calculated by the differential equation in (7) where $F_{\text {roll }}$ is calculated considering the mass of vehicle, bucket load and the equivalent mass of the wheels. The transferred force to the wheel is then calculated as follows:

$$
F_{w}=\frac{P_{t u r b}}{\eta_{g b} V}
$$

and finally the power required for traction is calculated only for 


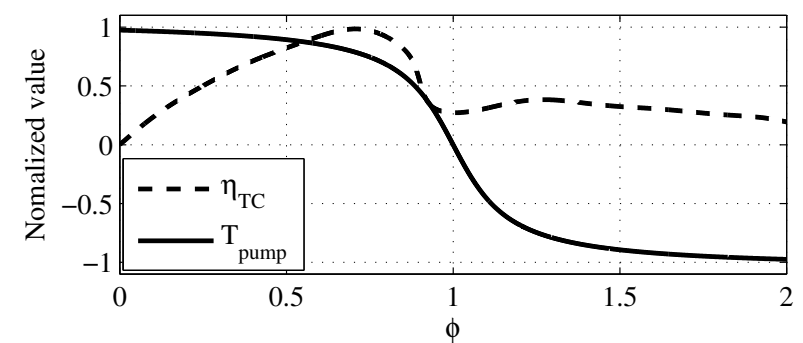

Figure 6: Normalized characteristic curves for TC efficiency and pumping torque.

the times that the gearbox is not in neutral according to:

$$
P_{\text {trac }}=P_{\text {pump }}|\operatorname{sign}(\gamma)|
$$

\subsubsection{Steering subsystem}

Steering system dynamics are described in exactly same manner as $<14>$ where the control input is the rate of change in the steering angle $u_{s}$ and the state variables are position in $X$ and $Y$ direction, steering angle $\delta$ and heading angle of the vehicle $\beta$ which are described by (10)-(11). The power required for WL steering is calculated to be proportional to steering angle dynamics as follows:

$$
P_{\text {steer }}=c_{s t} u_{s}^{2}
$$

\subsection{WL model fuel consumption validation}

To select the best values for the tuning parameters in the lifting, driveline and steering subsystems, exact values for the mass flow rate to the lifting and steering pumps or transferred power to the wheels are required. However these were not available in the measurements and therefore the tuning parameters are selected such that while steering angle and lifting height remain the same as a given measured cycle, following also the measured vehicle speed trajectory by the model result in almost same amount of fuel consumption as that of the measured cycle.

Iterating on the tuning parameter values and comparing the modeled versus measured fuel consumption for all available measured cycles, the tuning parameters are accepted when the determination coefficient $R^{2}=0.9357$ is achieved. This is a fairly close estimation of fuel consumption considering the simplifications made during the modeling. Figure 7 shows the calculated and measured fuel consumptions values.

\subsection{Optimal control problem formulation}

The developed model is used in OC problem formulations in order to calculate the trade-off between operation time and fuel consumption and corresponding WL trajectory in the SLC. In order to describe the boundary conditions of the OC problem the average of measured values are used as the starting and ending constraints on engine speed and lifting height.

Gearbox gear ratios are discontinuous control inputs to the WL model which make it difficult to solve the problem with common OC problem solving techniques. However, inspired by the performance of experienced operators during the experiments, the sequence of gears in SLC operation is decided as

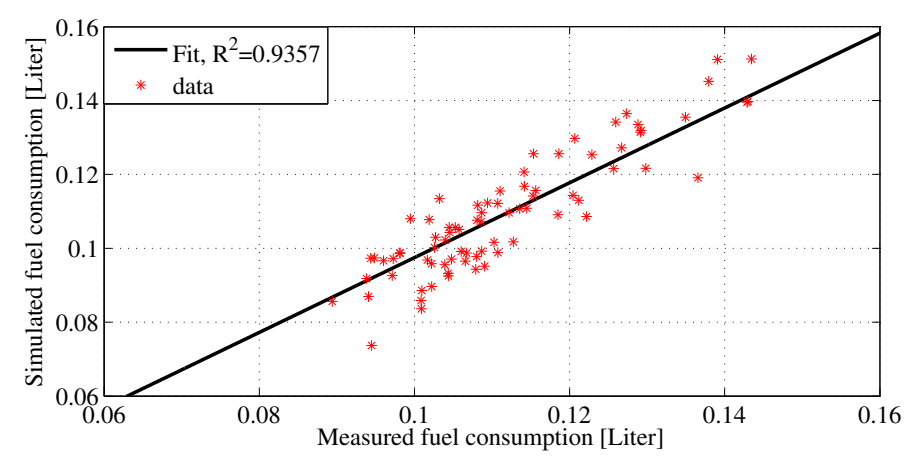

Figure 7: Comparison between simulated fuel consumption by the model and measured fuel consumption.

shown in Figure 1. WL operation between loading and unloading points of the SLC is then divided into four phases during which the gear ratio remains unchanged. Assuming $t=0$ [s] as the starting time of the SLC, ending time of each phase, $t=t_{1,2,3}$, and final time $t=T$ of the SLC operation are decision variables which need to be calculated by the OC problem solver in a way that the desired objective function is minimized, more details in $<14>$. This is a common approach enabling OC problem formulation in presence of integer control variables while it also reduces the problem dimension as one control input is removed from the formulations, see $<18>$ for details.

The optimal transients of the WL are calculated by solving OC problems of the following minimization form using the dynamics $f(x(t), u(t))$ described by (3)-(11):

$$
\begin{aligned}
\min _{x(t), u(t)} & w_{1} \times T+w_{2} \times m_{f} \\
\text { s.t. } & \\
\dot{x}(t) & =f(x(t), u(t), \gamma(t)) \\
w_{1}+w_{2} & =1 \\
u_{\text {min }} \leq u(t) & \leq u_{\text {max }} \\
x_{\text {min }} \leq x(t) & \leq x_{\text {max }} \\
R_{\text {turn }} & \leq R_{\text {turn,max }} \\
T_{e} & \leq T_{e, \text { max }} \\
\gamma(t) & =\gamma, \\
\gamma(t) & =0, \dot{v}(t) \leq \dot{v}_{\text {lim }}, \quad t \in \\
x(0) & \in x_{0} \\
x(T) & \in x_{T}, \dot{x}(T)=0
\end{aligned}
$$

$w_{1,2}$ in (25a) are the weight factors and solving for $w_{1}=0$ or $w_{2}=0$ gives the fuel optimal or time optimal transients, respectively, while the trade-off between fuel and time objectives can be calculated by solving for proper non-zero $w_{1,2}$. The constraints on controls, states, maximum engine torque $T_{e, \text { max }}$ and WL turning radius $R_{\text {turn,max }}$ are chosen according to $<16>$. The initial and final positions of the WL are set as $\left(X_{0}, Y_{0}\right)$ and $\left(X_{T}, Y_{T}\right)$ in Figure 3.

The mentioned constraints and the OC problem formulation are used when solving for the case which is referred to as free trajectory in the the following section. The prob- 
Table 1: Definition of constraints for special OC problem cases.

\begin{tabular}{|c|c|}
\hline Constraint & Description \\
\hline $\begin{array}{l}\text { fixed unloading orienta- } \\
\text { tion (fixed UO) }\end{array}$ & $\begin{array}{l}\beta(T)=\text { const }_{1} \text {, where } \text { const }_{1} \text { is set } \\
\text { equal to the value from a measured cy- } \\
\text { cle. }\end{array}$ \\
\hline constrained steering & $\begin{array}{l}\text { const }_{2} u_{s, \min } \leq u_{s}(t) \leq \text { const }_{2} u_{s, \text { max }} \text {, } \\
\text { where } \text { const }_{2} \in(0,1) \text { is selected such } \\
\text { that the maximum absolute steering } \\
\text { angle } \delta \text { becomes closer to a desired } \\
\text { measured cycle. }\end{array}$ \\
\hline trajectory $=$ specific path & $\begin{array}{l}X(Y)-e p s<X(t)<X(Y)+e p s, \\
\text { to follow a specific trajectory, path } \\
\text { constraints are set with the upper and } \\
\text { lower limits assigned according to ref- } \\
\text { erence polynomials. These polynomi- } \\
\text { als, defined in form of } X(Y) \text {, for re- } \\
\text { versing and forwarding sections of the } \\
\text { SLC are approximated using the mea- } \\
\text { sured data and curve fitting techniques. }\end{array}$ \\
\hline
\end{tabular}

lem is also solved for three special cases by including additional constraints into the problem formulation which are described in Table 1.

Due to model nonlinearities and the large number of state and control variables in the OC problem, instead of using classic OC problem solving approaches such as dynamic programming or Pontryagin maximum principle $<19>$, an OC solver named PROPT $<20>$ is used. This tool uses Pseudospectral methods $<21>$ for discretizing the states, control variables, objective function and constraints and then uses SNOPT $<22>$ to solve the resulting nonlinear programming problem. However, solving the OC problem in (25a)-(25k) with PROPT results in oscillatory optimal controls which are not desirable and the penalizing techniques described in $<23>$ are utilized.

\section{Results and discussion}

In this section, first the measurements are analyzed in terms of fuel consumption, operation time and choice of trajectory by the WL operators to find the properties that have the highest impact on the total productivity. The trade-off between fuel consumption and cycle duration is presented in the second section while candidate trajectories from the measurements are used as reference in OC problem formulation. The sensitivity of trajectories with respect to the orientation of WL at the unloading point and operators' steering speed is also analyzed.

\subsection{Measurements analysis}

As shown in Figure 3, the productivity cloud consists of two major group of points one with average low productivity at the bottom left and another containing the majority of points with higher average productivity. Checking transferred masses, the low productivity operations are found to be results of poor bucket filling as the amount of transferred load at those points is almost half of the others. It is also clear that trajectories with longer travel distance need more traction power which increases the fuel consumption and makes the longer trajectories less efficient. Nevertheless, there is still a considerable difference,

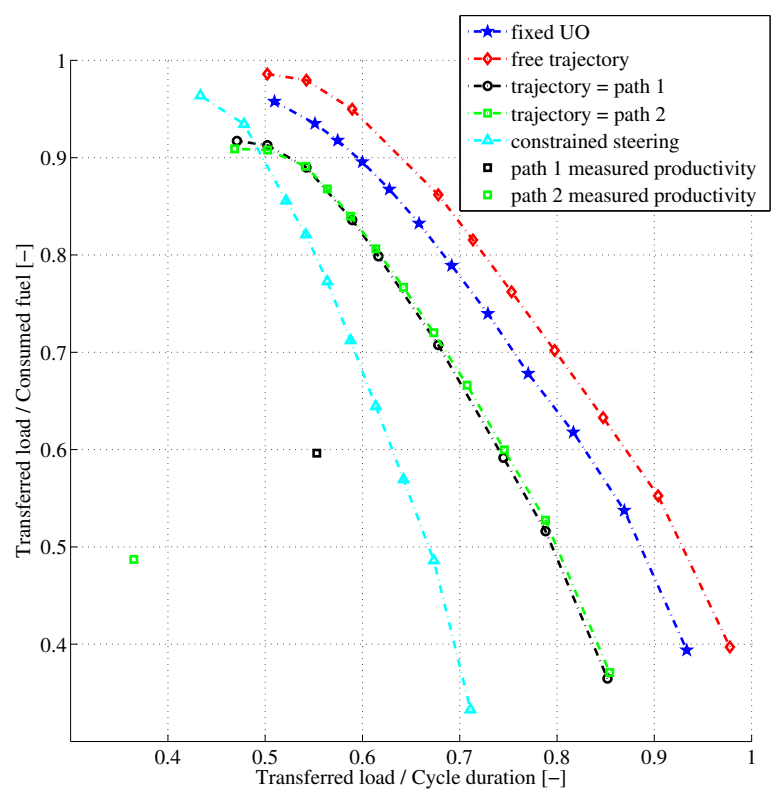

Figure 8: Four trade-offs are calculated for different trajectory properties by implementing the constraints in Table 1. Adding more limits on trajectory and forcing it away from optimal pushes the trade-off downwards. For confidentiality values are normalized with respect to maximums in this figure.

nearly $40 \%$, between the cycles in the higher productivity region while the bucket load or length of trajectory do not vary much between these points. For example, the difference between traveled distance and bucket load for the two selected trajectories shown on Figure 3 is only $1.5 \%$ and $1.2 \%$ respectively, however the operation in path 2 has taken $+53 \%$ longer time compared to path 1 . Fuel consumption is also $+23 \%$ higher in path 2 operation.

It can be concluded that productivity analysis based only on the comparison of the bucket load or travel distance is not reliable, and the duration of the operation is also important. This, from the modeling point of view, means that it is necessary to include main subsystems affecting both fuel consumption and duration of operation in the WL model. Fuel consumption depends on the diesel engine operation which in turn depends on the engine load relative to the power requirement in the power consumers, namely, the lifting and steering subsystems and vehicle traction. Describing the dynamics of the diesel engine and power consuming subsystems over time results in a time and load dependent WL model containing the necessary properties suitable for WL productivity analysis.

\subsection{Optimal control results analysis}

Figure 8 shows the calculated trade-offs between fuel and time for different trajectory constraints according to Table 1, and also the productivity corresponding to path 1 and path 2 from Figure 3. Figure 9 shows the corresponding trajectories plotted with same color as the trade-offs. The leftmost point on each trade-off is the fuel optimal solution and the rightmost point represents the time optimal solution while the rest of the points are calculated by choosing different weights 
$w_{1,2}$ in (25a). The points are connected by linear interpolation shown with the dashed-dotted line for better illustration. The start and end positions of the WL, set in $(25 \mathrm{j})$ and $(25 \mathrm{k})$, are identical for all trade-offs while same component restrictions as mentioned in OCP formulation are used in all cases. The free trajectory trade-off is calculated without applying any limiting constraint on the trajectory properties while by defining proper constraints on $X, Y$ and $\beta$ the path 1 and path 2 trade-offs are calculated with exact same trajectory and unloading orientation as path 1 and 2 in Figure 3. The fixed UO trade-off is calculated where the trajectory is free and only the orientation at unloading point is fixed to that of path 1 , and finally the constrained steering trade-off is calculated under same conditions as the free trajectory trade-off while multiplying the maximum allowed rate of change in steering angle $u_{s}$ with a reduction factor.

Considering the path 1 and path 2 trade-offs, it can be verified that the two trajectories have similar achievable productivity and the reason for different measured productivities in Figure 3 is the different engine and lifting transients. The highest productivity, free trajectory trade-off, is achieved when the trajectory is not limited and is obtained via optimization. As the trajectory gets more and more restricted in the fixed UO, path 1 and path 2 cases, the productivity decreases. The cycles on free trajectory trade-off have $23 \%$ shorter traveled distance compared to the selected path 1 and the gap between path 1-path 2 trade-offs and the free trajectory tradeoff shows that there is potentially place for up to $20 \%$ improvement of WL productivity in the faster operations. Same type of comparison between free trajectory and fixed UO tradeoffs shows that the trajectory is sensitive to disturbances and by changing the final orientation of the vehicle from optimal, the productivity reduces by almost $5 \%$ at all cycle durations.

Figure 9 shows that when unloading orientation is optimal, the trajectories at all cycle durations on the trade-off are more operator friendly since less steering effort is demanded. This is concluded by comparing the S-shape of the fixed UO trajectories between reversing point and unloading point against the almost circular free trajectory which is obtained by an almost constant steering wheel position. The traveled distance also increases by $6.8 \%$ in the case of having a non-optimal unloading orientation which increases both cycle duration and fuel consumption and consequently lowers the productivity of the operation.

General comparison between the measured trajectories in Figure 9 and OC results shows that WL operators mostly tend to start the operation by driving at least 5 [m] straight backward and then aim for the unloading point in the forwarding section of the SLC by changing the steering angle mostly during this section of the cycle. This means that the operators are reluctant to perform fast steering maneuvers specificity at the beginning. The operators are most likely not even capable of performing very fast because the start of the operation coincides with the initial lifting of the loaded bucket which demands high operator attention.

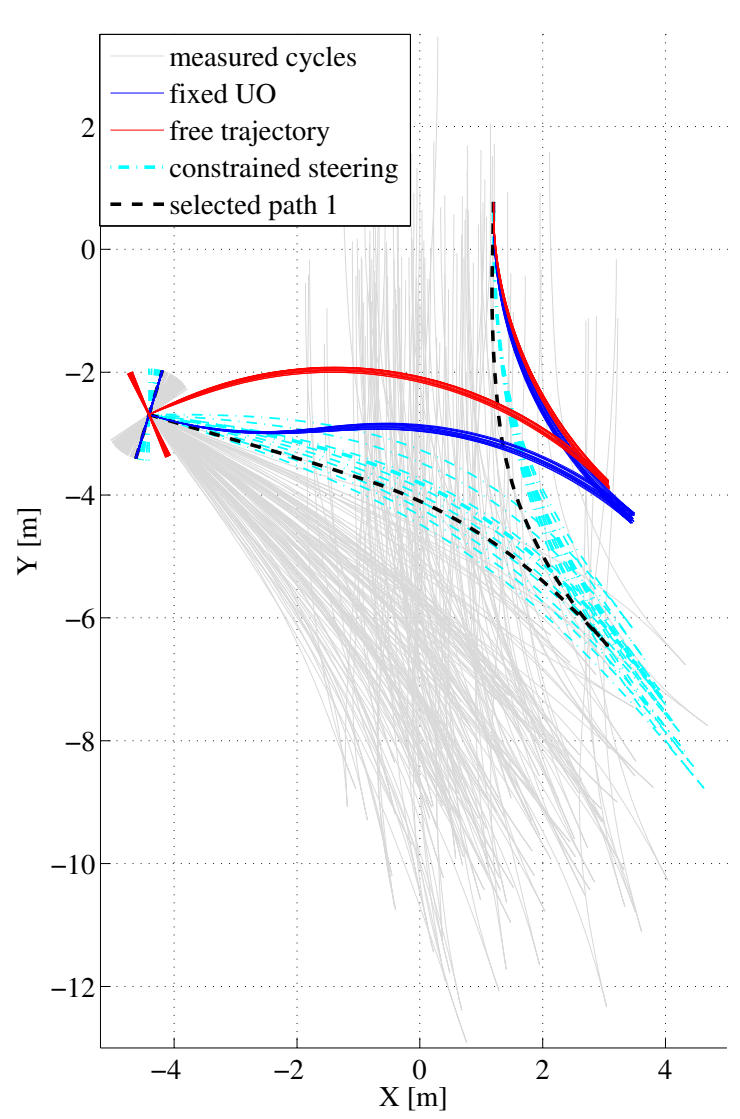

Figure 9: The trajectories between loading and unloading points in the SLC from measurements and $\mathrm{OC}$ results. Between free trajectory and fixed UO trajectories, the free trajectory ones are easier to follow by WL operators compared to the S-shaped fixed UO trajectories which are results of constrained unloading orientation (UO).

\subsubsection{Constrained steering}

To investigate how the productivity and trajectory change when the WL operator is not as fast as suggested by the optimal free trajectory, the maximum allowed steering speed $u_{s}$ is reduced according to Table 1 and the constrained steering trade-off and trajectories are calculated. Figure 10 shows $u_{s}$ and steering angle of the free trajectory, and the constrained steering case for a cycle duration close to path 1 and also the measured steering angle from path 1 . The slower steering speed reduces the productivity mainly because it increases the required time to align the vehicle along short and efficient trajectories which is why the constrained steering trade-off is squeezed to the left. This effect however is not identical on trajectories at all cycle durations and as the point on the trade-off moves closer to the fuel optimal side of the curve, the cycle time extends. There is better possibility to align the WL along the trajectories closer to the optimal solution; therefore, the deviation from the optimal red trajectory reduces as shown in Figure 9. It is also seen, in Figure 10 , that by limiting the rate of change in steering angle in the constrained steering case, the corresponding trajectory on Figure 9 becomes closer to path 1 . Considering the repetitiveness of WL operation in the short loading cycle and the suggested optimal steering transients, it is highly likely that 


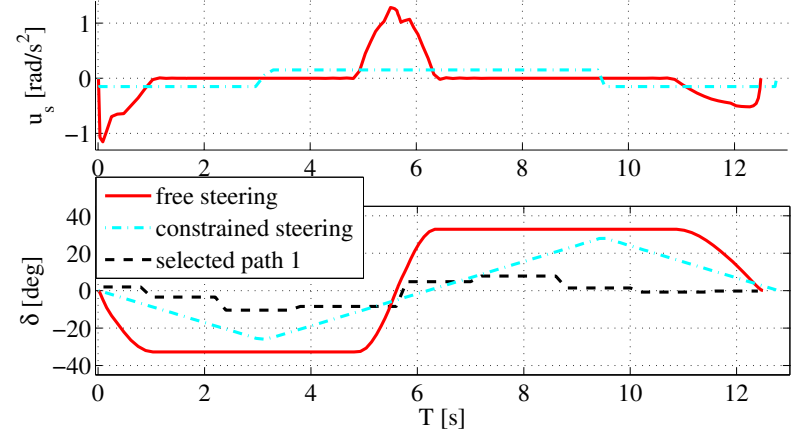

Figure 10: Comparison between optimal, constrained and measured steering dynamics. Steering speed control input (top) and steering angle (bottom).

a human operator, no matter how skilled, would not perform the steering with acceptable accuracy and speed over longer periods of time. The gap between human operator capability in WL steering and the requirements for optimal WL trajectories, can be narrowed by development and implementation of steering techniques such as autonomous vehicles.

\section{Conclusion}

Optimal control analysis of wheel loader operation in short loading cycle and comparison with measurements is carried out. An already developed wheel loader model is improved by better description of lifting dynamics and a improved torque converter model. The wheel loader model is then validated against measurements from real world operation, and it is shown that it can closely estimate the fuel consumption. Using the model in optimal control problem formulations and solving for various conditions, a side to side comparison is made between the measurements and the optimal control solutions.

It is shown that, although following an optimized trajectory is important, it does not guaranty high productivity and the productivity analysis in loading cycles has to be carried out in presence of all major power consuming sources such as vehicle dynamics, lifting and steering subsystems. It is crucial to consider the requirements of an efficient trajectory when deciding about working environment properties such as the available distance for the reversing maneuver and the orientation in which the load receiver vehicle is placed. It is also shown that that maintaining high rate of change in steering angle during the loading operation is important in order to follow efficient trajectories in a shorter time. However, human operators are likely to get tired and not react fast enough after certain time which encourages development of autonomous wheel loader systems.

The suggested approach for wheel loader operation analysis using optimal control is shown to be capable of describing the major phenomena during wheel loader operation and an efficient variant for such investigations.

\section{References}

[1] S. Dadhich, A survey in automation of earth-moving machines, Research report at Luleå University of Technology, Department of Computer Science, Electrical and Space Engineering.
[2] R. Fales, E. Spencer, K. Chipperfield, F. Wagner, A. Kelkar, Modeling and control of a wheel loader with a human-in-the-loop assessment using virtual reality, Journal of dynamic systems, measurement, and control 127 (3) (2005) 415-423.

[3] T. Nilsson, A. Fröberg, J. Åslund, Optimal operation of a turbocharged diesel engine during transients, SAE International Journal of Engines, SAE Paper: 2012-01-0711 5 (2) (2012) 571-578.

[4] R. Filla, Quantifying operability of working machines, Ph.D. thesis, Linkping UniversityLinkping University, Fluid and Mechatronic Systems, The Institute of Technology (2011).

[5] R. Filla, Operator and machine models for dynamic simulation of construction machinery, iSRN/Report code: LiU-Tek-Lic 2005:44 (2005).

[6] D. Carter, A. Alleyne, Load modeling and emulation for an earthmoving vehicle powertrain, in: Proceedings of American Control Conference, 2003, Vol. 6, IEEE, 2003, pp. 4963-4968.

[7] E. A. Prasetiawan, Modeling, simulation and control of an earthmoving vehicle powertrain simulator, Ph.D. thesis, University of Illinois at Urbana-Champaign (2001).

[8] R. Zhang, A. Alleyne, E. Prasetiawan, Modeling and H2/Ho MIMO control of an earthmoving vehicle powertrain, Journal of dynamic systems, measurement, and control 124 (4) (2002) 625-636.

[9] R. Filla, Optimizing the trajectory of a wheel loader working in short loading cycles, in: 13th Scandinavian International Conference on Fluid Power, SICFP2013, 2013, pp. 3-5.

[10] H. Takahashi, Y. Konishi, Path generation for autonomous locomotion of articulated steering wheel loader, Computer-Aided Civil and Infrastructure Engineering 16 (3) (2001) 159-168.

[11] S. Sarata, Y. Weeramhaeng, T. Tsubouchi, Approach path generation to scooping position for wheel loader, in: Robotics and Automation, 2005. ICRA 2005. Proceedings of the 2005 IEEE International Conference on, IEEE, 2005, pp. 1809-1814.

[12] S. Sarata, N. Koyachi, T. Tsubouchi, H. Osumi, M. Kurisu, K. Sugawara, Development of autonomous system for loading operation by wheel loader, in: Proceedings of the 23rd International Symposium on Automation and Robotics in Construction, 2006, pp. 466-471.

[13] B. Frank, A. Fröberg, Establishing an optimal work cycle for an alternative wheel loader concept, in: International Exposition for Power Transmission, IFPE 2014, Las Vegas, USA, 2014.

[14] V. Nezhadali, L. Eriksson, Optimal Lifting and Path Profiles for a Wheel Loader Considering Engine and Turbo Limitations, Vol. 455 of Lecture Notes in Control and Information Sciences. Editors: Harald Waschl and Ilya Kolmanovsky and Maarten Steinbuch and Luigi del Re, Springer International Publishing, 2014.

[15] B. Frank, L. Skogh, M. Alaküla, On wheel loader fuel efficiency difference due to operator behaviour distribution, in: 2nd International Commercial Vehicle Technology Symposium, CVT, 2012.

[16] Volvo, L220F wheel loader product brochure, "http://www . volvoce. com/constructionequipment/europe/en-gb/products/ wheelloaders/wheelloaders/L220F/Pages/specifications. aspx" (September 2014).

[17] A. Sciarretta, Vehicle propulsion systems: introduction to modeling and optimization, Springer, 2007.

[18] S. Sager, Reformulations and algorithms for the optimization of switching decisions in nonlinear optimal control, Journal of Process Control (2009) 1238-1247.

[19] L. S. Pontryagin, Mathematical theory of optimal processes, CRC Press, 1987.

[20] TOMLAB, PROPT - Matlab Optimal Control Software, "http://www . tomdyn. com/" (2014).

[21] G. Elnagar, M. A. Kazemi, M. Razzaghi, The pseudospectral legendre method for discretizing optimal control problems, Automatic Control, IEEE Transactions on 40 (10) (1995) 1793-1796.

[22] P. E. Gill, W. Murray, M. A. Saunders, Snopt: An sqp algorithm for largescale constrained optimization (2002).

[23] V. Nezhadali, L. Eriksson, Optimal control of wheel loader operation in the short loading cycle using two braking alternatives, in: 9th IEEE Vehicle Power and Propulsion Conference, IEEE VPPC 2013, Beijing, China, 2013. 